\title{
Computer-Aided Design of Intermittent Kinematics Mechanisms
}

\author{
Alexander Sobolev ${ }^{1,}$, , Alexei Nekrasov ${ }^{1}$, Michael Arbuzov ${ }^{1}$, and Alexei Rivkin ${ }^{1}$ \\ ${ }^{1}$ MSUT “STANKIN”, 1, Vadkovsky per., Moscow, 127994, Russia
}

\begin{abstract}
An overview of the application of intermittent kinematics mechanisms in modern technology is given. Approaches and methods of computer-aided design of intermittent kinematics mechanisms using CAD systems are considered. Based on the results of the performed automated modeling, the manufacture of intermittent kinematics mechanisms for use in the educational process of the university was carried out.
\end{abstract}

\section{Introduction}

The mechanisms of intermittent kinematics have found application in modern technology for the implementation of periodic stops of the movement of the output links with a constant rotational movement of the input links. The main types of such mechanisms are Maltese mechanisms and gear mechanisms of intermittent rotational movement. The Maltese Mechanism is an intermittent kinematics mechanism that converts the uniform rotational motion of the crank into intermittent rotational motion of the cross. The mechanism was mainly used in film projectors as a hopping mechanism for intermittent movement of film by a frame step. The creators of the Maltese movement in cinematography are considered the Frenchmen Constansuz and Bunzli, who received patent number 261292 on November 14, 1896 [1]. An alternative to the use of Maltese gears is the use of intermittent rotary gear mechanisms. As a rule, gear mechanisms consist of a driving incomplete gear with an involute tooth profile and a driven full-toothed cylindrical involute gear. Known sketches of gears by Leonardo da Vinci, containing incomplete gears [2].

\section{Application of intermittent kinematics mechanisms}

Maltese mechanisms have found application in modern technology [3-9]. Maltese mechanisms are used in modern machine tools. In particular, for periodic rotation of multiposition tables and drums, machine spindle blocks, turrets. The Maltese mechanisms were especially important in revolving heads [6]. The Maltese mechanism is used to rotate a target in a research facility to study the properties of elementary particles [7].

Maltese mechanisms are used in turnstiles and gates in smooth finishing devices $[8,9]$.

\footnotetext{
*Corresponding author: t-64@mail.ru
} 
Maltese mechanisms are used in the space industry in cameras for research stations. So on the American space probe "Dawn" the Maltese mechanism was used to replace filters in the framing camera to provide increased fault tolerance [10].

Maltese mechanisms are used in military equipment for the detection and video recording of air and ground objects, as well as in the field of active and passive location in order to increase the exposure time of the observed space.

Toothed mechanisms of intermittent rotational motion are used in metering devices, navigation devices, and kinematic travel limiters. In technological machines, they are used as drives of actuators of dividing mechanisms, intermittent rotation of the table of flexible production modules and intermittent feed of the conveyor in printing presses [3].

Advantages of intermittent kinematics gear mechanisms in comparison with the Maltese mechanisms:

- Large load capacity.

- The ability to change the laws of motion (cyclograms of work): the ratio of the time of motion and rest; angles of rotation of the driving and driven wheels. In the Maltese mechanisms, there is a constant law of motion of the cross with quasi-impacts at the beginning of the take-off run and the end of the run-out, which, along with an adverse effect on the dynamics of the mechanism, limits the effective use of balancing devices. The value of the relative duration of the rotation of the output shaft of the Maltese cross cannot be set arbitrarily in accordance with the cycle diagram of the machine, since it depends on the number of grooves of the cross.

The disadvantage of gear mechanisms of intermittent kinematics: the presence of collision of gear wheels at the beginning and end of the movement of the driven wheel. To fix the driven wheel at rest and mitigate its impact on the driving gear wheel at the moment of its entry and exit from engagement, special locking devices are provided in the mechanism design: locking arcs, sectors with tines or pin gears.

\section{Maltese Machinery Design Automation}

In order to accelerate the overall design process of mechanical engineering objects (mechatronic modules) containing Maltese mechanisms, the authors have developed a software application for automated calculation and construction of models of Maltese mechanisms in the T-Flex CAD design system based on API technology. A version of the software application for the design of Maltese mechanisms in the AutoCAD environment has also been developed.

The structure of the application is shown in Fig. 1. General view of the software application is shown in Fig. 2.

In fig. 3 shows the results of automatic creation of models of mechanisms in a CAD system using the developed software application.

The C \# Clipper library was used to calculate equidistant trajectories of the tool movement in a software application. On a special tab of the software application, the Gcode is generated for milling and EDM machines (Fig. 4).

To create control programs for CNC machines, the CAM module built into the T-Flex can also be used. The module uses the models that were generated by the software application we developed. An example of the display of the tool path during contour milling is shown in Fig. 5.

With the help of the developed software application, the calculation and manufacture of a real Maltese mechanism (Fig. 6) was carried out on a milling machining complex Awea BM 1100. This mechanism will be used in the educational process at the department of machine tools of MSUT "STANKIN" to educate students and improve their technical outlook. 


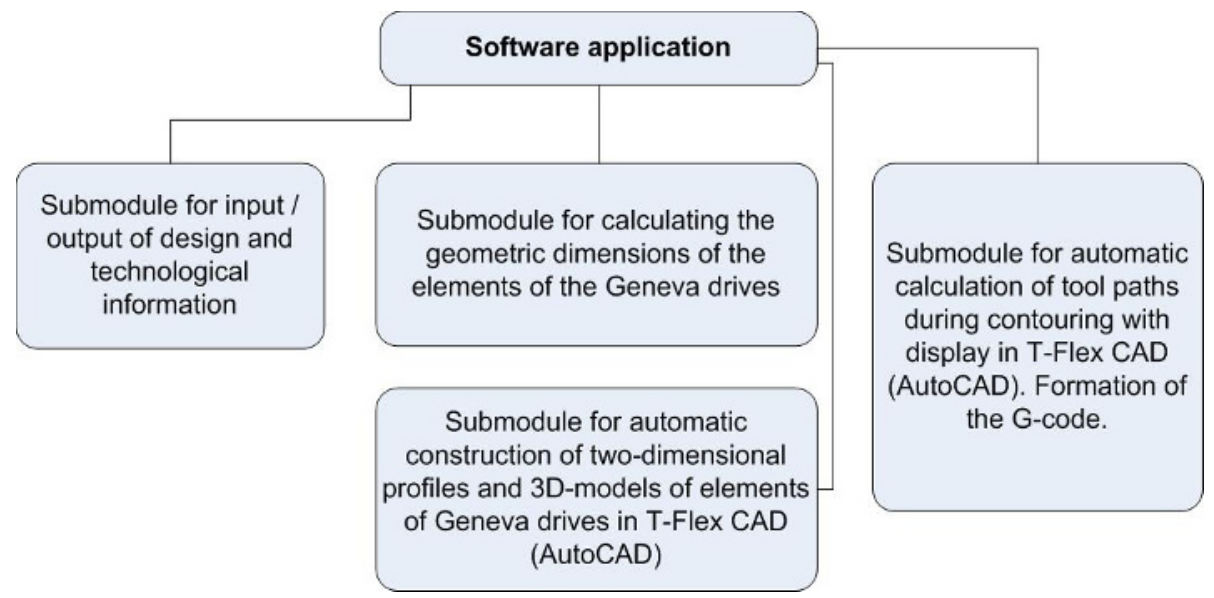

Fig. 1. The structure of the software application.

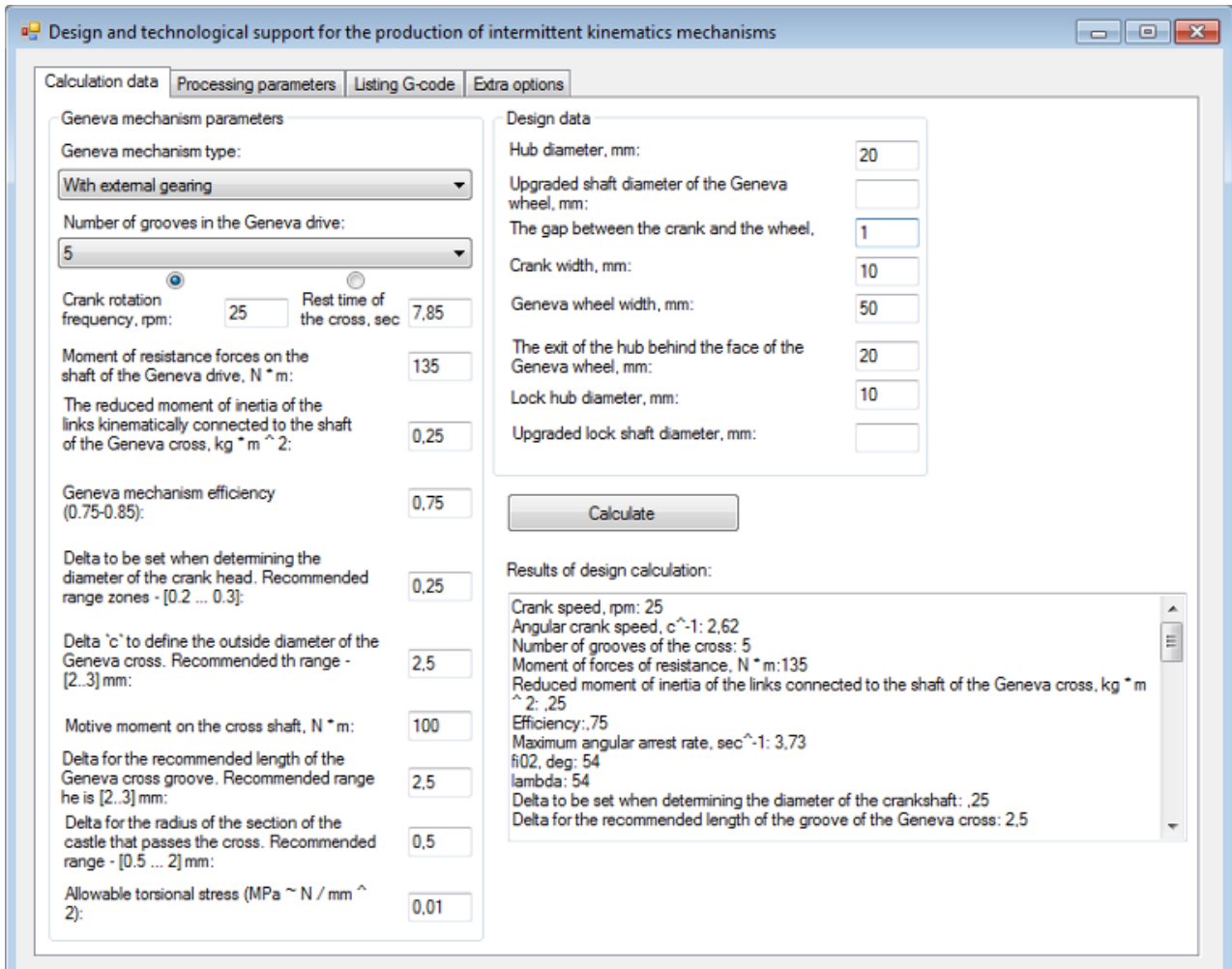

Fig. 2. General view of the software application. 


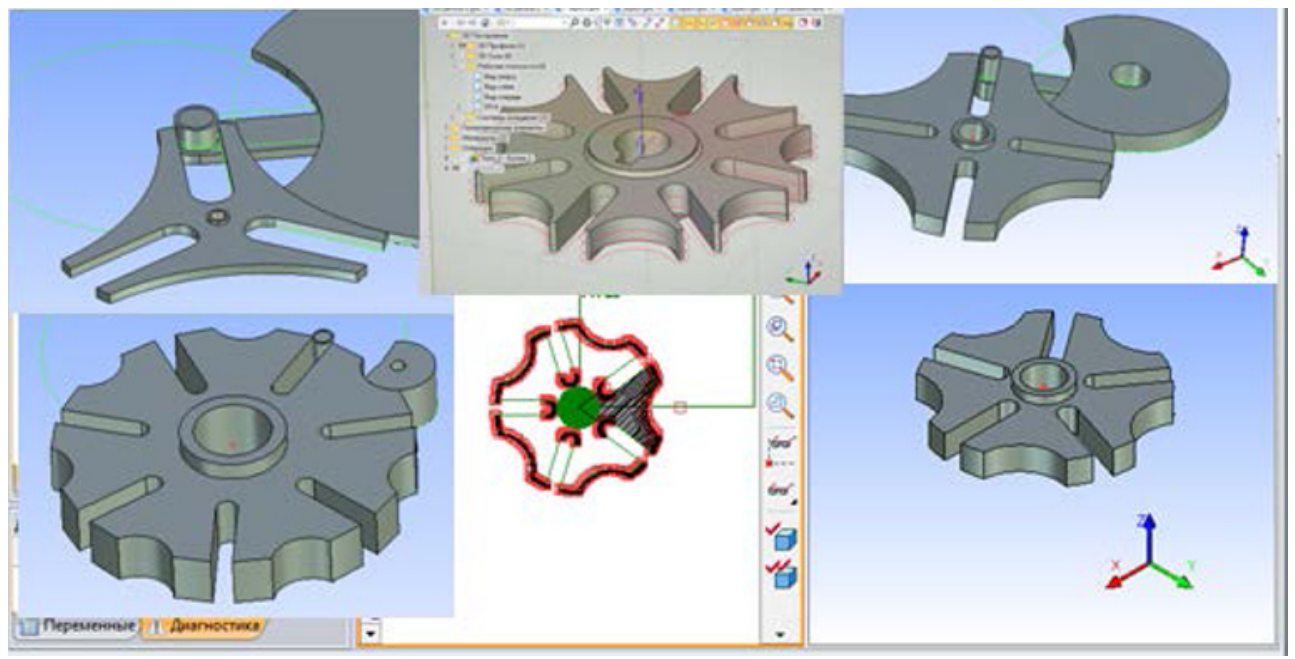

Fig. 3. Automatically generated models of Maltese movements in the CAD system.

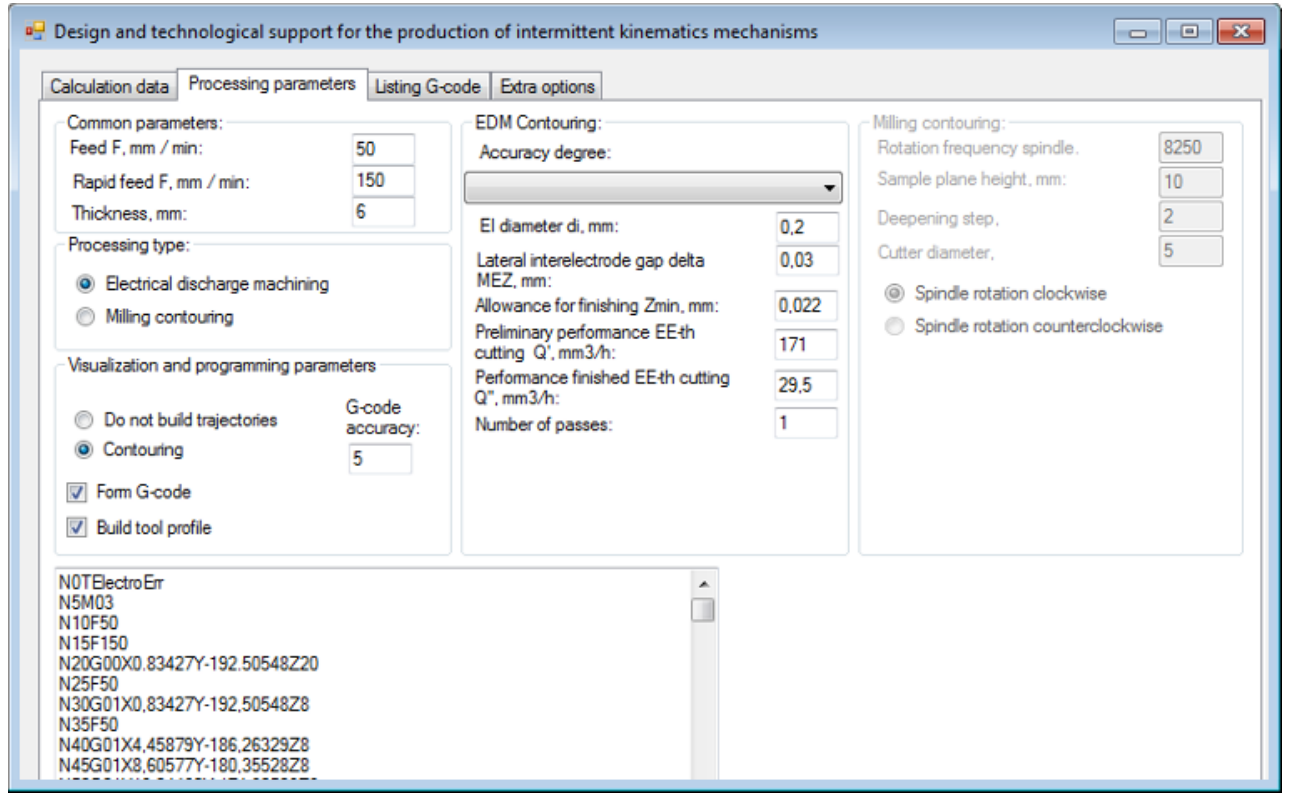

Fig. 4. Automatic generation of G-code in a software application. 


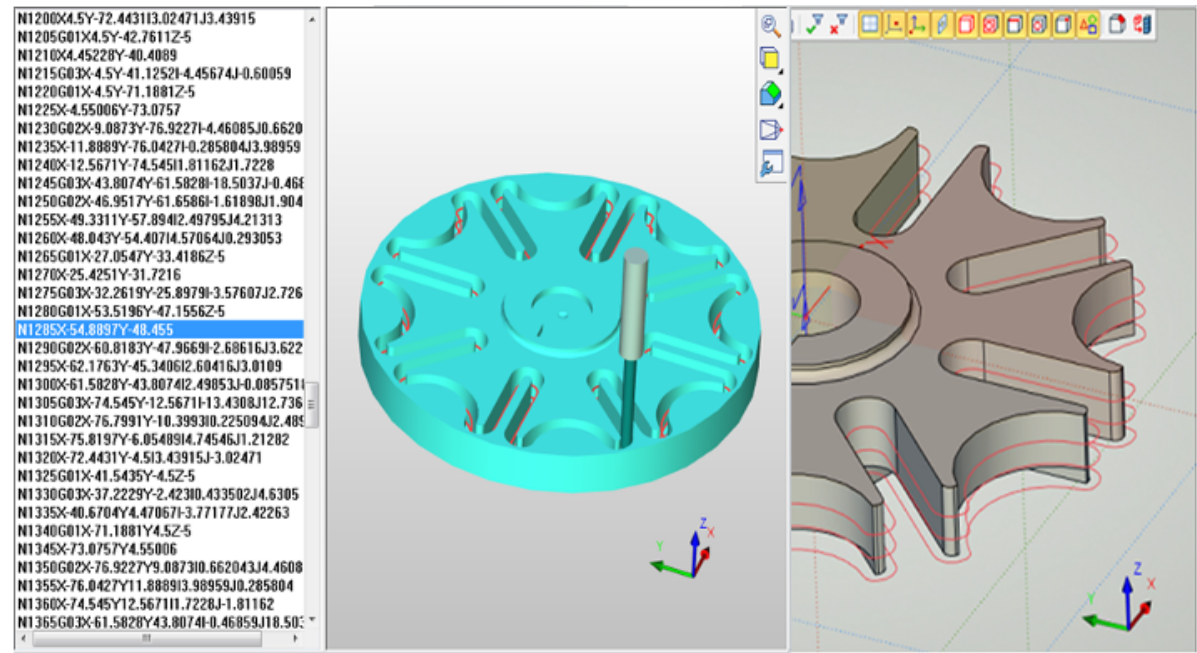

Fig. 5. The trajectories of the tool during manufacture Maltese cross.

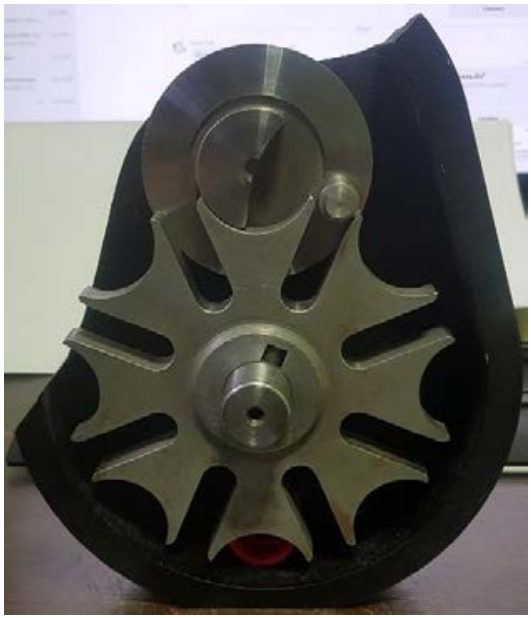

Fig. 6. Maltese mechanism made on a milling machining complex.

\section{Design automation of gear mechanisms of intermittent kinematics}

For automated calculation, profiling and modeling of gear mechanisms in CAD systems, the authors used their own software application described in the work [11].

The calculation of gear mechanisms of intermittent motion includes the determination of the gearing modulus, the geometric dimensions of the wheels and the cyclic characteristics of the mechanism. Algorithms for calculating the geometric dimensions of spur gears of an involute profile, implemented in a software application, are taken from [3, 4]. The calculation of the cyclic characteristics of mechanisms with incomplete gear wheels is carried out according to the method from work [3].

The planetary gear mechanism of intermittent kinematics is designed in the T-Flex CAD system (Fig. 7).

The input link of the mechanism (Fig. 7) will be the central full gear wheel, which transmits torque to the full gear satellite of the satellite block. The satellite block rotates as 
a whole. An incomplete gear wheel (satellite) of the block of satellites rolls around a stationary incomplete central (support) gear wheel and carries with it the carrier, which will be the output link providing the necessary cyclogram for the operation of the mechanism with intermittent movement. The movement of an incomplete satellite with one tooth, and, consequently, of the carrier is carried out during the entry of the satellite tooth into the cavity between the elements of the incomplete central gearwheel of internal gearing. At the moment of contact of the incomplete satellite and the wheel along the arcs, the full wheels engage with teeth and rotate, but the carrier is motionless. Replacement during the modernization of the central support wheel of internal gearing will change the output law of motion of the carrier as an output link.

Three-dimensional models of the mechanism parts from the CAD system were converted into STL format for their subsequent printing on a 3D printer using the Cura program. The $3 \mathrm{D}$ printer was assembled from purchased parts and assemblies, and the control system was built on the basis of an Arduino microcontroller.

The result of 3D printing is shown in Fig. 8. This mechanism will also be used in the educational process at the department of machine tools of MSUT "STANKIN".

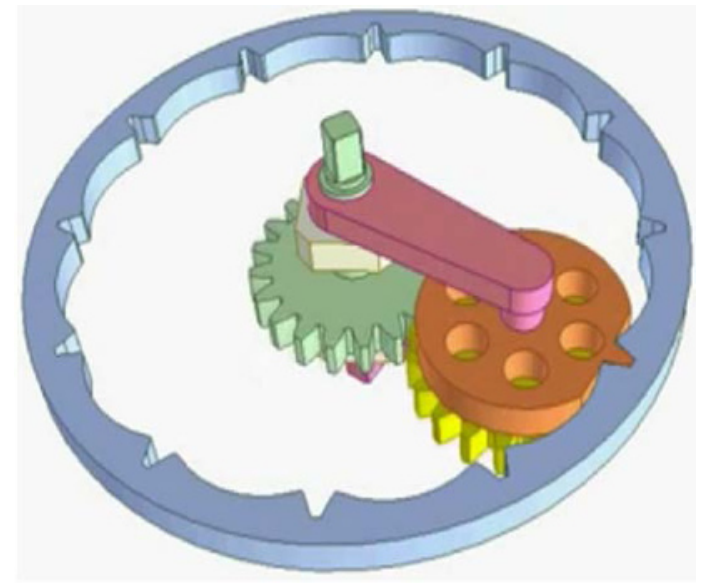

Fig. 7. Intermittent kinematics gear mechanism.

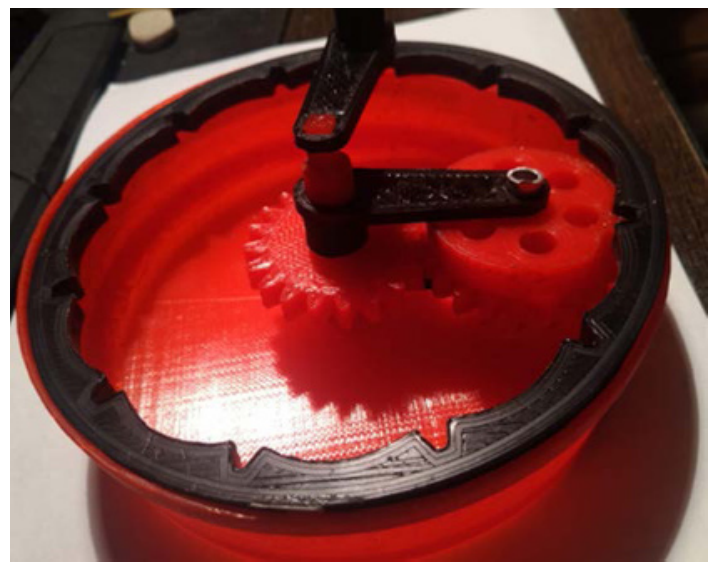

Fig. 8. 3D printed sample of the intermittent kinematics gear mechanism. 


\section{Conclusion}

The paper shows that the use of intermittent kinematics mechanisms in modern technology is still relevant. These mechanisms can be used in aggressive environments where the use of electronic modules of other types is difficult.

The authors have developed special software applications and computer-aided design techniques that significantly simplify the process of design and technological preparation of the production of elements of intermittent kinematics mechanisms using CAD systems.

The methods of computer-aided design developed by the authors were tested by manufacturing intermittent kinematics mechanisms on modern equipment of machinebuilding industries.

\section{References}

1. Wikipedia contributors, "Geneva drive", Wikipedia, The Free Encyclopedia, https://en.wikipedia.org/w/index.php?title=Geneva_drive\&oldid=1012689026 (accessed April 21, 2021)

2. M.V. Alferova, "Something about the mechanisms the Master used", WikiReading, https://biography.wikireading.ru/31690 (accessed April 21, 2021)

3. K.I. Zablonsky, I.M. Belokonev, B.M. Shchekin, The theory of mechanisms and machines (Vyshcha school, Kiev, 1989)

4. V.G. Gushchin, S.A. Baltadzhi, A.N. Sobolev, Yu.I. Brovkina, Design of mechanisms and machines (TNT LLC, Stary Oskol, 2020)

5. Y.I. Brovkina, A.N. Sobolev, A.Y. Nekrasov, Research of Characteristics and Parameters of Cycloidal Gear, Lecture Notes in Mechanical Engineering, pp.11691179 (2019)

6. A.N. Sobolev, A.Ya Nekrasov, CAD/CAE Modeling of Maltese Cross Mechanisms in Machine Tools, Russian Engineering Research, v. 36(4), pp. 300-302 (2016)

7. C. Haddock, B. Crawford, W.Fox, Slotted Rotatable Target Assembly Used In a Search for Possible Long Range Spin Dependent Interactions From Vector Boson Exchange Using Polarized Slow Neutron, Cornell University, https://arxiv.org/abs/1707.08303 (accessed April 21, 2021)

8. A.V. Andrianov, Turniket [Turnstile], Patent Rossiiskaia Federatsiia no. 2387782 (2009)

9. A.V. Andrianov, Kalitka [Gate], Patent Rossiiskaia Federatsiia no. 2538518 (2013)

10. C.T. Russell, C.A. Raymond, The Dawn Mission to Minor Planets 4 Vesta and 1 Ceres (Springer Science \& Business Media, 2012)

11. A.V. Rivkin, A.Ya. Nekrasov, A.N. Sobolev, M.O. Arbusov, The features of the gears profile forming by the electrical discharge machining, Materials Today: Proceedings, v. 38(4), pp. 1358-1363 (2021). 